\title{
Different subgroups regarding the absence of rescue therapy in intravenous immunoglobulin-resistant Kawasaki disease
}

\author{
Toshimasa Nakada ${ }^{1}$ \\ ${ }^{I}$ Department of Pediatrics, Aomori Prefectural Central Hospital
}

\begin{abstract}
The objective of this study is the description of clinical outcomes regarding the absence of rescue therapy of intravenous immunoglobulin (IVIG)-resistant Kawasaki disease. I investigated the clinical outcomes of 33 IVIG-resistant patients who received the initial single IVIG therapy with the delayed use of antiinflammatory drugs (DUA). These patients were divided into two groups: 11 patients who received rescue therapies (rescue group) and 22 patients who did not receive rescue therapy (non-rescue group). One patient of rescue group developed coronary artery lesions (CAL). None of the patients of non-rescue group developed CAL. The prevalence of persistent fever between rescue and non-rescue groups at 3 days and 4 days after initial IVIG therapy was $100 \%$ vs. $77 \%(P=0.144)$ and $100 \%$ vs. $18 \%(P<0.001)$, respectively. The C-reactive protein value, neutrophil counts, serum albumin levels, and sodium levels were significantly different between two groups at median 3 days after initial IVIG therapy. Two-thirds of the IVIG-resistant patients diagnosed at 24 $\mathrm{h}$ after completion of the initial IVIG therapy with DUA did not develop CAL after 30 days of illness without rescue therapies. The rescue therapy at this time may lead to overtreatment.
\end{abstract}

Keywords - Kawasaki disease, Coronary artery lesions, Intravenous immunoglobulin therapy, Immunoglobulinresistance, $C$-reactive protein

\section{INTRODUCTION}

Kawasaki disease is an acute systemic vasculitis of unknown cause that mainly affects infants and children [1]. Coronary artery lesions (CAL) are one of the most important complications of this disease, and an intravenous immunoglobulin (IVIG) resistance is one of the most important factors for CAL development during the acute phase of Kawasaki disease. However, the clinical outcomes of IVIG-resistant Kawasaki disease have not been established.

The recent Japanese guidelines for the acute-phase treatment of Kawasaki disease recommended the initiation of the rescue therapies at $24 \mathrm{~h}$ after completion of the initial IVIG therapy [2]. However, a recent study showed that fever in the first $36 \mathrm{~h}$ following the initial IVIG therapy completion was not predictive of CAL [3]. This study recommended refraining from rescue therapies until $36 \mathrm{~h}$ after completion of the initial IVIG therapy. Another recent study defined the IVIG-resistant patients as those with persistent fever who failed to achieve defervescence within $48 \mathrm{~h}$ after completion of the initial IVIG therapy [4]. The description of the clinical outcomes of the IVIG-resistant patients may lead to the appropriate timing of the rescue therapies for these patients.

Recent studies have disclosed that aspirin and flurbiprofen appeared to have a negative impact on the suppressive effects of initial IVIG therapy for CAL development during the acute phase of Kawasaki disease and that an initial single IVIG therapy dose with the delayed use of anti-inflammatory drugs (DUA) might be effective for CAL suppression [5,6]. The clinical outcomes of the IVIG-resistant patients who received the initial IVIG therapy with DUA remain unclear.

The hypotheses of this study were that a subgroup of the IVIG-resistant patients diagnosed at $24 \mathrm{~h}$ after completion of the initial IVIG therapy with DUA don't develop CAL after 30 days of illness without rescue therapies and that the rescue therapy at this time may lead to overtreatment.

The objective of this study is the description of clinical outcomes of IVIG-resistant Kawasaki disease after initial IVIG therapy with DUA and clarification of useful cut-off values of laboratory tests for rescue therapies.

\section{METHODS}

This retrospective study included 144 consecutive patients who had received an initial $2 \mathrm{~g} / \mathrm{kg} / \mathrm{dose}$ of IVIG therapy with DUA (aspirin or flurbiprofen) for Kawasaki disease between January 2004 and September 2015 at the Department of Pediatrics, Aomori Prefectural Central Hospital. Anti-inflammatory drugs were initiated within $24 \mathrm{~h}$ after the end of initial IVIG infusion [5]. The diagnosis of Kawasaki disease was based on Japanese Criteria (Fifth Edition) [7]. 
In this study, recurrence and relapse were defined differently. When Kawasaki disease recurred after an initial disappearance of the major symptoms and improvement in the test results, it was defined as a recurrence. If there is an interval of at least two months from the onset of the first Kawasaki disease illness to onset the new episode, it was defined as recurrence [8]. If a child became afebrile during the acute phase, an exacerbation or reappearance of major symptoms without other pyrogenic disease was defined as a relapse. Defervescence was defined as body temperature $<37.5^{\circ} \mathrm{C}$ for $24 \mathrm{~h}$, and the time of the defervescence was defined as when the body temperature became $<37.5^{\circ} \mathrm{C}$. IVIG-resistance was defined if fever persisted or had reappeared at $24 \mathrm{~h}$ after first-line treatment [2].

I excluded 29 patients who received concomitant IVIG and anti-inflammatory therapy, four patients who had developed CAL before the start of therapy, and seven patients with disease recurrence and one patient who developed left ventricular dysfunction because of their greater prevalence of CAL $[9,10,11]$.

The 33 IVIG-resistant patients were divided into two groups: a rescue group, comprising 11 patients who received the rescue therapies for the initial IVIG therapy resistance, and a non-rescue group, comprising 22 children who did not receive the rescue therapy for the initial IVIG resistance.

The Egami score, which is the risk score for prediction of IVIG-resistance using the clinical findings including age, illness days, platelet count, alanine aminotransferase, and C-reactive protein (CRP), was evaluated before initial IVIG therapy [12].

2.1. Anti-inflammatory Drugs Therapy and Initial IVIG Therapy

The choice between aspirin and flurbiprofen was made by each doctor after considering the patient's liver function and the risk of Reye syndrome during the influenza pandemic. Flurbiprofen had been used in cases of severe aspirin hepatotoxicity in Japan and Reye syndrome is not mentioned as the adverse effects and precautions of flurbiprofen [13]. Flurbiprofen was more commonly used before 2009 because of advantage for hepatic dysfuntion and Reye syndrome. Aspirin was more commonly used after 2009 because aspirin use was a global standard for Kawasaki disease. Aspirin was initiated at a dose of $30 \mathrm{mg} / \mathrm{kg} / \mathrm{day}$ and decreased to $5-10$ $\mathrm{mg} / \mathrm{kg} /$ day when the patients became afebrile. Flurbiprofen was initiated at a dose of $3-5 \mathrm{mg} / \mathrm{kg} / \mathrm{day}$ and decreased to $3 \mathrm{mg} / \mathrm{kg} / \mathrm{day}$ when the patients became afebrile.

During the study period, an initial IVIG regimen of $2 \mathrm{~g} / \mathrm{kg} / \mathrm{dose}$ starting on day 5 of the illness was used as first-line therapy when possible. The regimen of the initial IVIG therapy with DUA was used after 2004. A part of patients received this therapy with DUA between 2004 and 2008. The choice between DUA and the concomitant use of anti-inflammatory drugs was made by each doctor during this period. After 2009, the initial IVIG therapy with DUA was used for all patients [5]

2.2. Rescue Therapy

The decision for rescue therapies in resistant patients was made between 48 and $72 \mathrm{~h}$ after the end of the initial IVIG therapy. The decision was made comprehensively according to clinical parameters, including body temperature, major symptoms of Kawasaki disease, general condition, and laboratory data. There were no definite cut-off values of laboratory tests during this study.

The blood samples were taken 2-4 days after initial IVIG therapy and the samples just before decision were used for study. CRP ratio was defined as ratio of CRP values after/before initial IVIG therapy [14].

Second-line therapy was rescue IVIG therapy, and third-line therapy was ulinastatin infusion. Plasma exchange was adopted after 2014 as another third-line therapy option. This regimen has been approved in Aomori Prefectural Central Hospital [14, 15]. Written informed consent was obtained from the parents or guardians of all children before the initial therapy $[5,6]$.

2.3. Diagnosis of CAL

CAL was diagnosed by echocardiography as follows [16]. CAL was diagnosed when any of these examinations showed an internal lumen diameter $\geqq 3 \mathrm{~mm}$ in a patient $<5$ years of age or a diameter $\geqq 4 \mathrm{~mm}$ in a patient $\geqq 5$ years of age; if the internal diameter of a segment was at least 1.5 times as large as that of an adjacent segment; or if the lumen appeared irregular. Transient CAL was defined as the disappearance of CAL within 30 days of the illness.

Myocardial ischemia due to CAL is one of the most important complications caused by Kawasaki disease. Long-term follow-up studies have shown that a maximum CAL size $>5 \mathrm{~mm}$ was a statistically significant predictive risk factor for myocardial ischemia, and that all CAL $\leq 5 \mathrm{~mm}$ in size regressed to normal size [17]. Another study reported that the threshold diameter for acute phase CAL that developed into subsequent stenosis was $6.0 \mathrm{~mm}$ [18]. Therefore, the prevention of CAL of $>5 \mathrm{~mm}$ may be an important goal in the acute treatment of Kawasaki disease to prevent coronary artery stenosis in later stages of the disease [19]. In this study, CAL were evaluated by $\mathrm{mm}$ for the entire population because of the goal to the prevention of coronary artery stenosis in later stages.

2.4. Statistical Analysis 
Statistical analyses were performed with StatFlex Version 6 for Windows (Artech Co., Ltd., Osaka, Japan). Chi-square test, Fisher's exact test, and Mann-Whitney U test were used as appropriate with a specific sample size calculation. A value of $P<0.05$ was considered statistically significant.

\section{RESULTS}

Thirty-three among 144 patients $(23 \%)$ were resistant to the initial IVIG therapy. The 33 patients included 17 boys and 16 girls. The median age was 2 years 10 months (range, 3 months-12 years 4 months).

The severity of Kawasaki disease before initial IVIG therapy, as evaluated using the Egami score [12], was significantly different between the two groups. The patients of the rescue group were more severely ill than those of the non-rescue group (Table 1). Sex, age of onset, the prevalence of incomplete type, were not significantly different between the two groups (Table 1). The laboratory findings except leukocyte count were not significantly different between the two groups before initial IVIG therapy (Table 1). The leukocyte count of the non-rescue group was significantly higher than that of the rescue group (Table 1).

Table 1. Comparison of the clinical findings and laboratory values before initial IVIG therapy between rescue

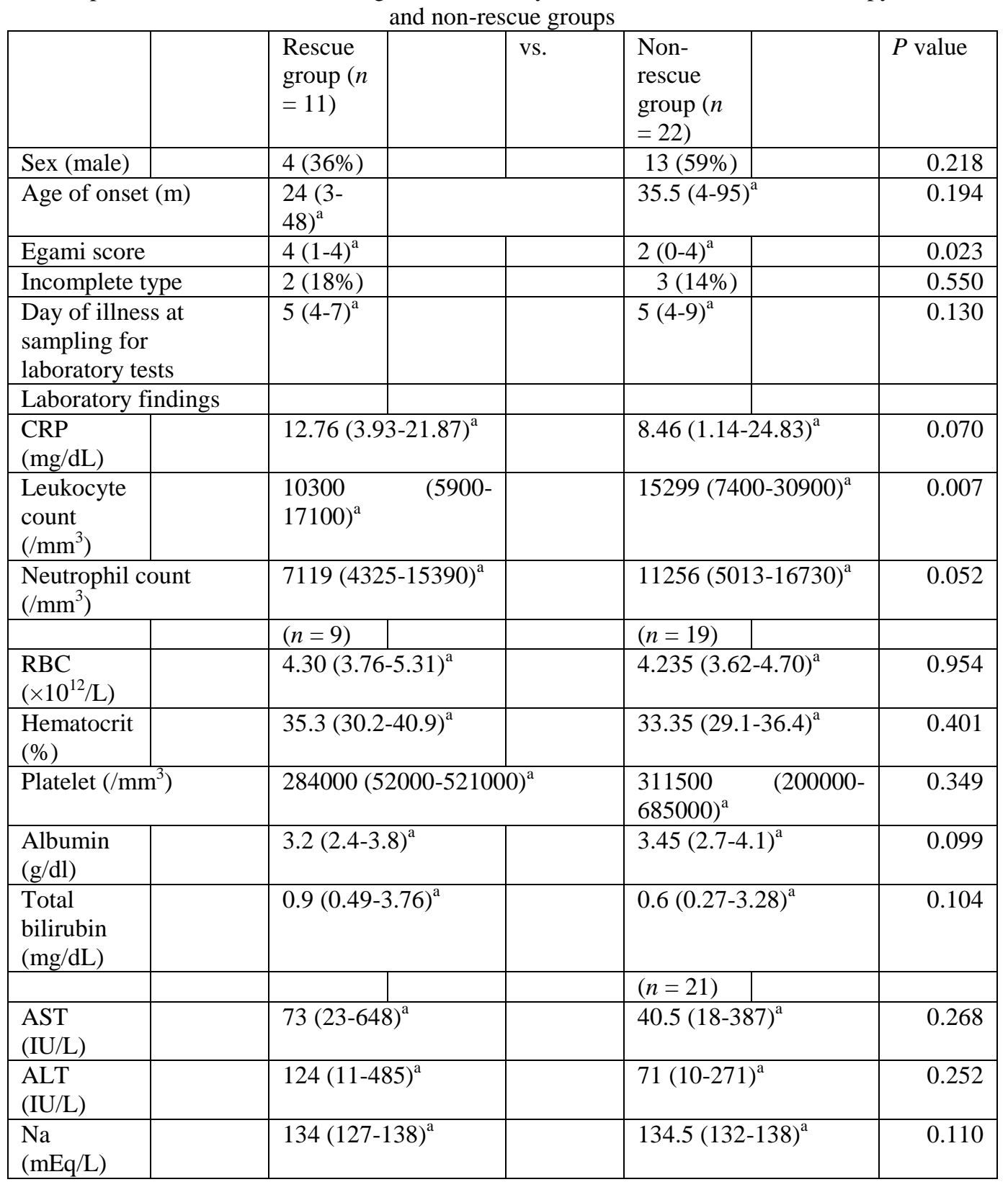

a: median (minimum-maximum ); Incomplete type: patients with fewer than four major symptoms; IVIG: 
intravenous immunoglobulin; CAL: coronary artery lesions; CRP: C-reactive protein; RBC: red blood cell count; AST: aspartate aminotransferase; ALT: alanine aminotransferase; Na: serum sodium.

The prevalence of the type of anti-inflammatory drugs (aspirin or flurbiprofen), and the start day of the initial IVIG therapy were not significantly different between the two groups (Table 2).

Table 2. Comparison of the treatment and laboratory information after initial IVIG therapy between rescue and non-rescue groups

\begin{tabular}{|c|c|c|c|c|c|c|}
\hline & & $\begin{array}{l}\text { Rescue } \\
\text { group }(n \\
=11)\end{array}$ & vs. & $\begin{array}{l}\text { Non- } \\
\text { rescue } \\
\text { group }(n= \\
22)\end{array}$ & & $P$ value \\
\hline \multicolumn{7}{|l|}{ Initial IVIG } \\
\hline \multicolumn{2}{|c|}{ Start day of illness } & $5(4-7)^{\mathrm{a}}$ & & $5(5-9)^{\mathrm{a}}$ & & 0.162 \\
\hline Aspirin & & $4(36 \%)$ & & $11(50 \%)$ & & \\
\hline Flurbiprofen & & $7(64 \%)$ & & $11(50 \%)$ & & 0.712 \\
\hline \multicolumn{7}{|l|}{ Defervescence } \\
\hline \multicolumn{2}{|l|}{ Day of illness } & $\begin{array}{l}11(9- \\
16)^{\mathrm{a}}\end{array}$ & & $8(7-12)^{\mathrm{a}}$ & & 0.001 \\
\hline \multicolumn{2}{|c|}{$\begin{array}{l}\text { Duration from start of initial IVIG } \\
\text { therapy (days) }\end{array}$} & $6(4-9)^{\mathrm{a}}$ & & $3(2-5)^{\mathrm{a}}$ & & $<0.001$ \\
\hline \multicolumn{7}{|c|}{ Patients with persistent fever } \\
\hline & $\begin{array}{l}2 \text { days after initial } \\
\text { IVIG }\end{array}$ & $\begin{array}{l}11 \\
(100 \%)\end{array}$ & & $22(100 \%)$ & & 1.000 \\
\hline & $\begin{array}{l}3 \text { days after initial } \\
\text { IVIG }\end{array}$ & $\begin{array}{l}11 \\
(100 \%)\end{array}$ & & $17(77 \%)$ & & 0.144 \\
\hline & $\begin{array}{l}4 \text { days after initial } \\
\text { IVIG }\end{array}$ & $\begin{array}{l}11 \\
(100 \%)\end{array}$ & & $4(18 \%)$ & & $<0.001$ \\
\hline & $\begin{array}{l}5 \text { days after initial } \\
\text { IVIG }\end{array}$ & $6(55 \%)$ & & $1(5 \%)$ & & 0.003 \\
\hline & $\begin{array}{l}6 \text { days after initial } \\
\text { IVIG }\end{array}$ & $6(55 \%)$ & & $0(0 \%)$ & & $<0.001$ \\
\hline & $\begin{array}{l}7 \text { days after initial } \\
\text { IVIG }\end{array}$ & $4(36 \%)$ & & $0(0 \%)$ & & 0.008 \\
\hline \multicolumn{7}{|c|}{ Laboratory findings } \\
\hline \multicolumn{2}{|c|}{ Days after IVIG } & $3(2-4)^{\mathrm{a}}$ & & $3(2-4)^{\mathrm{a}}$ & & 0.718 \\
\hline \begin{tabular}{l|l} 
CRP & \\
$(\mathrm{mg} / \mathrm{dL})$
\end{tabular} & & \multicolumn{2}{|c|}{$9.33(2.86-20.58)^{\mathrm{a}}$} & \multicolumn{2}{|c|}{$4.23(0.23-9.88)^{\mathrm{a}}$} & 0.003 \\
\hline CRP ratio & & \multicolumn{2}{|c|}{$0.76(0.53-1.39)^{\mathrm{a}}$} & \multicolumn{2}{|c|}{$0.47(0.20-1.13)^{\mathrm{a}}$} & 0.001 \\
\hline \multirow[t]{2}{*}{$\begin{array}{l}\text { Leukocyte } \\
\text { count } \\
\left(/ \mathrm{mm}^{3}\right) \\
\end{array}$} & & \multicolumn{2}{|c|}{$\begin{array}{l}11500 \quad(5100- \\
20600)^{\mathrm{a}}\end{array}$} & \multicolumn{2}{|c|}{$9500(4600-19000)^{\mathrm{a}}$} & 0.258 \\
\hline & & & & $(n=21)$ & & \\
\hline \multirow{2}{*}{\multicolumn{2}{|c|}{ Neutrophil count $\left(/ \mathrm{mm}^{3}\right)$}} & \multicolumn{2}{|c|}{$9545(1530-16274)^{\mathrm{a}}$} & \multicolumn{2}{|c|}{$5672(1449-9500)^{\mathrm{a}}$} & 0.029 \\
\hline & & & & $(n=20)$ & & \\
\hline \multirow[t]{2}{*}{$\begin{array}{l}\mathrm{RBC} \\
\left(\times 10^{12} / \mathrm{L}\right)\end{array}$} & & \multicolumn{2}{|c|}{$3.9(3.31-4.58)^{\mathrm{a}}$} & \multicolumn{2}{|c|}{$4.1(3.66-4.8)^{\mathrm{a}}$} & 0.100 \\
\hline & & & & $(n=21)$ & & \\
\hline \multirow[t]{2}{*}{$\begin{array}{l}\text { Hematocrit } \\
(\%)\end{array}$} & & \multicolumn{2}{|c|}{$31.8(26.3-37.1)^{\mathrm{a}}$} & \multicolumn{2}{|c|}{$32.2(30.6-40.6)^{\mathrm{a}}$} & 0.204 \\
\hline & & & & $(n=21)$ & & \\
\hline Platelet $\left(/ \mathrm{mm}^{3}\right)$ & & $\begin{array}{l}293000 \\
558000)^{\mathrm{a}}\end{array}$ & $(131000-$ & $\begin{array}{l}380000 \\
687000)^{\mathrm{a}}\end{array}$ & $(227000-$ & 0.071 \\
\hline
\end{tabular}


Different subgroups regarding the absence of rescue therapy

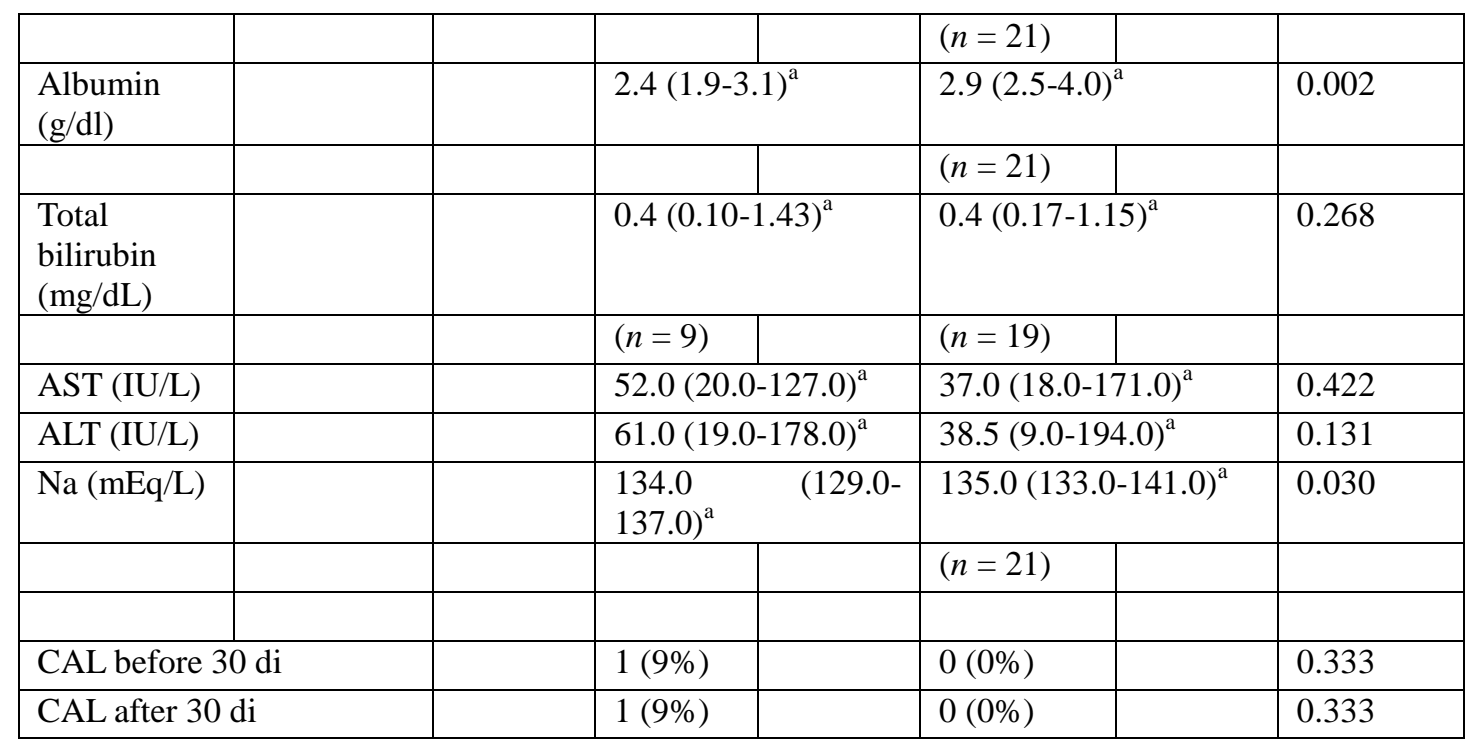

a: median (minimum-maximum ); IVIG: intravenous immunoglobulin; CAL: coronary artery lesions; CRP: Creactive protein; RBC: red blood cell count; AST: aspartate aminotransferase; ALT: alanine aminotransferase; $\mathrm{Na}$ : serum sodium.

C-reactive protein ratio was defined as ratio of CRP values after/before initial IVIG therapy.

All of 11 patients in the rescue group received the rescue IVIG therapies at median 9th (range, 7th11th) day of illness for the initial IVIG therapy resistance and duration between the first and second IVIG therapies were median 3 (3-4) days. Two of 11 patients received the third-line therapy; one patient received ulinastatin infusion, and another patient received the plasma exchange. Among the patients that received a thirdline therapy, one patient received corticosteroids after the ulinastatin infusion because of prolonged fever and intractable arthralgia [20]. Ten of 11 patients in rescue group received two doses of $2 \mathrm{~g} / \mathrm{kg} / \mathrm{dose}$ IVIG therapies. One patient who received plasma exchange received a third dose of IVIG therapy as fourth-line therapy [20]. None of the patients in rescue group received the rescue therapy for relapse of the disease.

None of the patients in the non-rescue group received rescue therapies for resistance and all of these patients recovered by themselves without further treatment for resistance. Only one patient in the non-rescue group received the rescue IVIG therapy for relapse of the disease after 13 days from initial IVIG therapy.

The day of illness to day of defervescence in the rescue group was more than that in the non-rescue group (Table 2). The duration from the start of initial IVIG therapy to defervescence in the rescue group was significantly longer than that of the non-rescue group (Table 2). The prevalence of the patients with persistent fever after initial IVIG therapy in the rescue group was significantly higher than that in the non-rescue group after 4 days from the start of initial IVIG therapy (Table 2). The prevalence of CAL before and after day 30 was similar between the two groups (Table 2).

The patient with CAL in the rescue group had CAL on day 8 , and this 2 -year-old girl received a plasma exchange on day 9 at the hospital of Hirosaki University School of Medicine for 3 days. Her CAL diameters of right proximal artery were $4.8 \mathrm{~mm}$ and $2.9 \mathrm{~mm}$ on day 21 and 40 of her illness, respectively. However, echocardiography on day 52 of illness showed the regression of CAL and normal internal coronary artery size. The selective coronary arteriogram performed at 7 months after the disease onset revealed no abnormal findings. Thereafter, she had been followed up without medication. Her clinical course had been uneventful at 1 year 3 months after the disease onset.

The time of the laboratory findings in the two groups were median 3 days after the start day of the initial IVIG therapy, and the times were similar between the two groups (Table 2). The following laboratory findings after initial IVIG therapy were significantly different between the rescue and non-rescue groups: CRP levels, CRP ratio, neutrophil counts, serum albumin levels, and serum sodium levels (Table 2).

The CRP ratio cut-off value of $<0.50$ excluded all 11 patients in the rescue group and included 13 nonrescue group patients of 33 IVIG-resistant patients (39\%).

\section{DISCUSSION}

This study identified the two-thirds of the patients who were diagnosed as IVIG-resistant at $24 \mathrm{~h}$ after the completion of the initial IVIG infusion and did not have associated CAL after 30 days of illness without the rescue therapies. This finding is consistent with the results of the recent study; fever in the first $36 \mathrm{~h}$ following 
the initial IVIG therapy completion was not predictive of CAL, although all patients diagnosed with IVIG resistance in this study received rescue therapy [3].

The establishment of the appropriate rescue therapies is important for suppression of CAL and for safety of the treatment in the acute phase of Kawasaki disease. This study showed that the clinical outcomes of the two subgroups in the IVIG-resistant patients were significantly different and that these two groups could be separated at 3 to 4 days after initial IVIG therapy.

The data regarding Egami score, defervescence, and laboratory tests suggested that the patients of the rescue group were more severely ill than those of the non-rescue group (Table 1,2). However, the prevalence of CAL were similar between the two groups (Table 2). These findings suggested that the rescue therapies at 3 to 4 days after initial IVIG therapy were effective for the suppression of CAL in the rescue group patients. On the other hand, none of the patients in the non-rescue group received rescue therapies for resistance and all of these patients recovered by themselves without further treatment for resistance. This finding suggested that the decision of rescue therapies at 3 to 4 days after initial IVIG therapy was appropriate for the patients in the nonrescue group.

The appropriate decision for rescue therapies leads to the appropriate use of IVIG and to the avoidance of the risk of rescue therapies. The recent 22nd nationwide survey of Kawasaki disease in Japan showed that $16 \%$ of patients received the rescue IVIG therapy for initial IVIG therapy resistance and that $3 \%$ of patients had associated CAL after 30 days of illness [21]. In this study, the prevalence of rescue IVIG therapy for initial IVIG therapy resistance was $8 \%$ (11/144), and the prevalence of CAL after 30 days and after one year of illness were $1 \%(1 / 144)$ and $0 \%$, respectively. These findings also suggested that the decision at 3 to 4 days after the start of initial IVIG therapy might be useful for the safe and effective rescue therapies for suppression of CAL.

Not only IVIG therapy-resistant patients but also IVIG therapy-responsive patients developed CAL caused by Kawasaki disease [20,22]. This means that the pathway of fever development may not be completely consistent with that of CAL development. Therefore, the factors except body temperature should be considered at the decision for rescue therapy. These factors may be general condition and laboratory findings.

The previous study showed the usefulness of CRP and neutrophil count for the decision for rescue therapies [23]. These factors were also significantly different between the rescue and non-rescue groups in this study. Furthermore, the CRP ratio was useful for the detection of the non-rescue group patients; $39 \%$ of the nonrescue group patients were detected from the IVIG-resistant patients by using CRP ratio. The previous study showed the usefulness of the CRP for prediction of the clinical course after initial IVIG therapy and CAL development among IVIG-resistant patients [4, 14, 24, 25]. The CRP ratio may be useful for determining unnecessary rescue therapies for the prevention of CAL after 30 days of illness. Critical decision for the appropriate rescue therapies can prevent the risk caused by the therapies and unnecessary use of IVIG. Currently, IVIG therapy, corticosteroid, infliximab, and plasma exchange have been used for rescue therapy. However, these therapies have the risk of anaphylaxis, possible development of CAL, infusion-associated reaction, and shock, respectively $[2,26]$. The refraining from corticosteroid use might be one of the factors for the favorable outcomes of CAL in this study [26].

The optimum start time of the initial IVIG therapy for the prevention of large CAL of internal diameter $>5 \mathrm{~mm}$ caused by Kawasaki disease has not been established. An epidemiological study in 15,940 patients in Japan showed that receipt of initial IVIG therapy before day 5 of the illness was significantly associated with IVIG non-response and CAL [22]. Another study reported that there was no evidence that IVIG therapy on day 4 or earlier had greater efficacy in preventing cardiac sequelae than therapy on days 5-9, and that early treatment would more likely require rescue IVIG therapy [27]. The favorable outcome of CAL and the low prevalence of rescue IVIG in this study showed that the start time on day 5 was not too late to prevent large CAL caused by Kawasaki disease.

\section{CONCLUSIONS}

Two-thirds of the IVIG-resistant patients diagnosed at $24 \mathrm{~h}$ after completion of the initial IVIG therapy with DUA did not develop CAL after 30 days of illness without rescue therapies. The rescue therapy at this time may lead to overtreatment.

The data regarding Egami score, defervescence, and laboratory tests suggested that the patients of the rescue group were more severely ill than those of the non-rescue group. However, the prevalence of CAL were similar between the two groups. These findings suggested that the rescue therapies at 3 to 4 days after initial IVIG therapy were effective for the suppression of CAL in the rescue group patients. On the other hand, none of the patients in the non-rescue group received rescue therapies for resistance and all of these patients recovered by themselves without further treatment for resistance. This finding suggested that the decision for rescue therapies at 3 to 4 days after initial IVIG therapy was appropriate for the patients in the non-rescue group. Three to 4 days after initial IVIG therapy with DUA may be appropriate for the decision for rescue therapies for IVIGresistant patients with Kawasaki disease. 
The limitation of this study was the small number of patients. The efficacy and safety of the strategy of this study should be investigated by large size study.

\section{ACKNOWLEDGMENTS}

I would like to thank the pediatric cardiologists of Hirosaki University School of Medicine for providing clinical information regarding the patient who received plasma exchange at the hospital of Hirosaki University School of Medicine, and all those who were involved in the medical management of the patients included in this study. There are no conflicts of interest to declare.

\section{REFERENCES}

[1] Burns JC, Glod é MP. Kawasaki syndrome, Lancet, 364, 2004, 533-544.

[2] Research committee of the Japanese Scociety of Pediatric Cardiology; Cardiac Surgery committee for development of guidelines for medical treatment of acute Kawasaki disease. Guidelines for medical treatment of acute Kawasaki disease: report of the Research committee of the Japanese Society of Pediatric Cardiology and Cardiac Surgery (2012 revised version), Pediatr Int, 56, 2014, 135-158.

[3] Jaggi P, Wang W, Dvorchik I, et al. Patterns of fever in children after primary treatment for Kawasaki disease, Pediatr Infect Dis J, 34, 2015, 1315-1318.

[4] Nakagama Y, Inuzuka R, Taiyu H, et al. Fever pattern and C-reactive protein predict response to rescue therapy in Kawasaki disease, Pediatr Int, 58, 2016, 180-184.

[5] Nakada T. Effects of anti-inflammatory drugs on intravenous immunoglobulin therapy in the acute phase of Kawasaki disease, Pediatr Cardiol, 36, 2015, 335-339.

[6] Ito H, Kiyosawa N. Effectiveness of aspirin therapy in acute phase of Kawasaki disease, Prog Med, 22, 2002, 1640-1643.

[7] Ayusawa M, Sonobe T, Uemura S, et al. Revision of diagnostic guidelines for Kawasaki disease (the $5^{\text {th }}$ revised edition), Pediatr Int, 47, 2005, 232-234.

[8] Hirata S, Nakamura Y, Yanagawa H. Incidence rate of recurrent Kawasaki disease and related risk factors: from the results of nationwide surveys of Kawasaki disease in Japan, Acta Paediatr, 90, 2001, 40-44.

[9] Nakamura Y, Yanagawa H, Ojima T, et al. Cardiac sequelae of Kawasaki disease among recurrent cases, Arch Dis Child, 78, 1998, 163-165.

[10] Yang H, Du ZD, Fu P. Clinical features of recurrent Kawasaki disease and its risk factors, Eur J Pediatr, 172, 2013, 1641-1647.

[11] Yoshikawa H, Nomura Y, Masuda K, et al. Four cases of Kawasaki syndrome complicated with myocarditis, Circ J, 70, 2006, 202-205.

[12] Egami K, Muta H, Ishii M, et al. Prediction of resistance to intravenous immunoglobulin treatment in patients with Kawasaki disease, J Pediatr, 149, 2006, 237-240.

[13] Japanese Circulation Society Joint Research Group. Guidelines for diagnosis and management of cardiovascular sequelae in Kawasaki disease, Pediatr Int, 47, 2005, 711-732.

[14] Nakada T. Usefulness of $\mathrm{C}$ - reactive protein for indication diagnosis of acute phase additional therapy in Kawasaki disease, Med J Aomori, 60, 2015, 1-6.

[15] Nakada T. Difference in the prevalence of coronary arterial lesions in Kawasaki disease according to the time of initiation of additional aspirin or flurbiprofen therapy, Med J Aomori, 57, 2012, 15-19.

[16] Kobayashi T, Inoue Y, Takeuchi K, et al. Prediction of intravenous immunoglobulin unresponsiveness in patients with Kawasaki disease, Circulation, 113, 2006, 2606-2612.

[17] Mueller F, Knirsch W, Harpes P, et al. Long-term follow-up of acute changes in coronary artery diameter caused by Kawasaki disease: risk factors for development of stenotic lesions, Clin Res Cardiol, 98, 2009, 501-507.

[18] Tsuda E, Kamiya T, Ono Y, et al. Incidence of stenotic lesions predicted by acute phase changes in coronary arterial diameter during Kawasaki disease, Pediatr Cardiol, 26, 2005, 73-79.

[19] Nakada T. Prevention of large coronary artery lesions caused by Kawasaki disease, Medical Research Archives (3), 2015, 1-7[DOI:http://dx.doi.org/10.18103/mra.v0i3.138]

[20] Nakada T. Background factors associated with the complications of coronary artery lesions caused by Kawasaki disease, Clinical Medicine Research, 4, 2015, 127-131.

[21] Makino N, Nakamura Y, Yashiro M, et al. Descriptive epidemiology of Kawasaki disease in Japan, 2011-2012: from the results of the $22^{\text {nd }}$ nationwide survey, J Epidemiol, 25, 2015, 239-245.

[22] Uehara R, Belay ED, Maddox RA, et al. Analysis of potential risk factors associated with nonresponse to initial intravenous immunoglobulin treatment among Kawasaki disease patients in Japan, Pediatr Infect Dis J, 27, 2008, 155-160. 
[23] Kim HK, Oh J, Hong YM, et al. Parameters to guide retreatment after initial intravenous immunoglobulin therapy in Kawasaki disease, Korean Circ J, 41, 2011, 379-384.

[24] Zhang CY, Liu LL, Liao Y, et al. Plasma prealbumin and C-reactive protein predicts coronary artery lesions in children with Kawasaki disease, Beijing Da Xue Xие Bao, 45, 2013, 207-210.

[25] Iwashima S, Kimura M, Ishikawa $\mathrm{T}$, et al. Importance of C-reactive protein level in predicting nonresponse to additional intravenous immunoglobulin treatment in children with Kawasaki disease, Clin Drug Investig, 31, 2011, 191-199.

[26] Zhao CN, Du ZD, Gao LL. Corticosteroid therapy might be associated with the development of coronary aneurysm in children with Kawasaki disease, Chinese Medical Journal, 129, 2016, 922-928.

[27] Muta H, Ishii M, Egami K et al. Early intravenous gamma-globulin treatment for Kawasaki disease: the nationwide surveys in Japan, J Pediatr, 144, 2004, 496-499. 